\title{
Adherence to Antiretroviral Therapy Among HIV-Infected Drug Users: A Meta-Analysis
}

\author{
Monica Malta $\cdot$ Monica M. F. Magnanini • \\ Steffanie A. Strathdee $\cdot$ Francisco I. Bastos
}

Published online: 20 November 2008

(C) Springer Science+Business Media, LLC 2008

\begin{abstract}
We conducted a meta-analysis of studies assessing adherence to highly active antiretroviral therapy (HAART) and a qualitative systematic review of factors associated with better HAART outcomes among HIV+ drug users (DU). Thirty-eight studies were considered, which analyzed 14,960 patients $(11,394 \mathrm{HIV}+\mathrm{DU}$, $76.2 \%$ ). Overall adherence (pooled percent of DU classified as adherent in each study) was 0.60 (95\% CI: $0.52-$ $0.68)$, similar to levels identified by studies conducted with $\mathrm{HIV}+$ patients who are not drug users. Time frame used to measure adherence was an independent predictor of interstudy heterogeneity. The systematic review identified better HAART outcomes among former DU, those with less severe psychiatric conditions, those receiving opioid substitution therapy and/or psychosocial support. Patients initiating HAART with lower viral load and higher CD4
\end{abstract}

M. Malta ( $\square)$

Social Science Department, Sergio Arouca School of Public Health (DCS/ENSP), Oswaldo Cruz Foundation,

Rua Leopoldo Bulhoes, 1480, Room 905, Manguinhos,

Rio de Janeiro, RJ 21041-210, Brazil

e-mail: momalta@cict.fiocruz.br; mmalta@jhsph.edu

M. M. F. Magnanini

Institute of Public Health Studies, Federal University

of Rio de Janeiro (IESC/UFRJ), Rio de Janeiro, Brazil

\section{S. A. Strathdee}

Division of International Health and Cross Cultural Medicine, Department of Family and Preventive Medicine at University of California, San Diego (UCSD) School of Medicine, San Diego, CA, USA

\section{F. I. Bastos}

Health Information Laboratory, Center for Scientific and Technological Information (LIS/ICICT), Oswaldo Cruz Foundation, Rio de Janeiro, Brazil counts, and those without co-infections also had better treatment outcomes. Our findings suggest that HIV+ DU tend to be inappropriately assumed to be less adherent and unlikely to achieve desirable treatment outcomes, when compared to their non-DU cohort.

Keywords HIV · AIDS - Adherence - Drug use · Meta-analysis

\section{Introduction}

Highly active antiretroviral therapy (HAART) has improved the health and quality of life of people living with HIV/AIDS (PLWHA) since its introduction in 1996/7. HAART reduces HIV viral load and increases CD4+ cell levels, delaying progression to AIDS and reducing mortality (Egger et al. 2002). Adherence to HAART is closely tied to HIV viral suppression and CD4 cell count response (Wood et al. 2003a, 2004a, b) and is a key predictor of antiretroviral success and survival (Hogg et al. 1998; Palella et al. 1998).

Since reductions in HIV viral load may significantly reduce HIV transmission (Wood et al. 2008), HAART appears to be an important addition to the arsenal of HIV prevention strategies (Quinn et al. 2000). However, since the advent of HAART, widening disparities in health outcomes among HIV-seropositive populations indicate the need to elucidate factors facilitating and impeding effective HAART use among disadvantaged or marginalized populations (Mills et al. 2006; Wood et al. 2003a).

Previous studies have found that active drug users had sub-optimal adherence, resulting in poorer virologic and immunologic outcomes to HAART compared to former and non-drug users (Lucas et al. 2001, 2002; Palepu et al. 
2003a, b). However, other studies have found that HIVpositive drug users who have access to drug abuse and mental health treatment (Malta et al. 2008), and particularly former drug users who are abstinent, can attain the same levels of adherence found among PLWHA who have never used illicit drugs (Crystal et al. 2001) and can thus achieve similar survival rates compared to non-drug using populations (Wood et al. 2008).

Drug users (DU) living with HIV/AIDS have been found to experience suboptimal access to HAART (Celentano et al. 1998; Bassetti et al. 1999; Strathdee et al. 1998), and tend to initiate HAART at more advanced stages of infection (Celentano et al. 2001; Wang et al. 2004). This raises concern since injection drug use is a major vector for HIV transmission in seven of 10 regions around the world (Mathers et al. 2008; WHO/UNAIDS 2007). Non-injecting DU are also at high risk for HIV infection due to high risk sexual behaviors (Inciardi and Surrat 2001; Latkin et al. 2001). In recent years, the number of PLWHA increased substantially in east and central Asia, as well as in eastern Europe, where nearly two-thirds of HIV infections (62\%) are attributed to injection drug use (Mathers et al. 2008). In highly populated countries such as China and India, injection drug use is also a major contributor to local HIV/ AIDS epidemics (WHO/UNAIDS 2007; Mathers et al. 2008).

Some providers prescribing HAART to DU have been concerned that clinical improvement may lead to high risk behaviors; patients learning that they have an undetectable viral load may believe he is no longer infectious and may start practicing unsafe sex (Atkinson et al. 2008; Vlahov and Celentano 2006). Others have been concerned that suboptimal adherence may favor the emergence of viral resistance and the transmission of drug-resistant HIV strains (Vlahov and Celentano 2006; Wainberg and Friedland 1998; Wensing et al. 2005).

The specific barriers that jeopardize HIV-positive drug users' adherence to HAART remain to be further elucidated. A former review paper by our group summarized the main barriers and facilitators of adherence among drug using populations (Malta et al. 2008). In this meta-analysis, we synthesize the available scientific literature, presenting a pooled measurement of adherence among HIV-positive DU accounting for between-study heterogeneity. We also summarize factors associated with optimal HIV viral suppression and/or immunological reconstitution among the subset of studies that examined these treatment outcomes.

\section{Methods}

In planning our recent systematic review (Malta et al. 2008) and meta-analysis, we reviewed standard guidelines to conduct and report meta-analysis studies, which included the consolidated standards of reporting trialsCONSORT (Altman et al. 2001; Moher et al. 2001), the quality of reporting of meta-analyses-QUOROM (Moher et al. 2000), the meta-analysis of observational studies in epidemiology (MOOSE) group (Stroup et al. 2000), and the transparent reporting of evaluations with nonrandomized designs-TREND (Des Jarlais et al. 2004). Since many of the selected studies were observational studies rather than clinical trials, the MOOSE recommendations were used to conduct and report the meta-analysis, while the TREND checklist (Version 1.0) was used as a guide for data abstraction.

\section{Search Strategy}

Search strategies were developed using systematic automated and manual searches. First, we conducted a comprehensive automated search of five electronic bibliographic databases-including MEDLINE via PubMed, Cochrane CENTRAL, AIDSLINE, AMED, CINAHL, TOXNET, SciELO, and ISI-Web of Science were searched from 1996 to February 29, 2008; except for AIDSLINE, which was searched from 1996 up to 2000, when the inclusion of new citations was discontinued. This search combined standardized search terms (keywords and medical subject heading terms) that reflect key domains: (a) HIV/AIDS, (b) compliance (i.e., adherence, compliance, directly observed therapy), (c) addiction (i.e., substance abuse, substance dependence), and (d) target population (i.e., PLWHA). Citations that intersect all four domains were downloaded into the study database.

To reduce publication bias and gaps in the automated search, we implemented four supplementary search strategies to identify additional studies. First, we searched the published conference abstracts from HIV/AIDS and STD conferences using the same domains as the automated search. Second we searched the National Institutes of Health's Computer Retrieval of Information on Scientific Projects (CRISP) database (http://crisp.cit.nih.gov) to identify researchers working in the field of HIV/AIDS and/ or drug addiction. Third, we contacted authors of selected papers to obtain upcoming publications. Finally, we reviewed the reference lists of all selected studies for additional citations. All studies identified through these procedures that met our eligible criteria were entered into the study database.

To be included in the review, studies had to evaluate adherence to HAART as the primary outcome, report a given cut-off to define optimal adherence (e.g., 90 or 95\%), include multivariable analyses to assess correlates of adherence and present data stratifying participants according to history of drug use (drug users vs. never drug 
users), or evaluate only current/former drug users on their studies. Further details are available elsewhere (Malta et al. 2008). Studies with an outcome defined as continuous were included in the systematic review (Malta et al. 2008), but not in the meta-analysis, due to methodological constraints associated with combining studies using continuous and discrete outcomes.

\section{Study Selection and Data Extraction}

Using a predefined protocol, two investigators (MM, FIB) extracted data from peer-reviewed papers addressing adherence among HIV-positive DU and independently assessed their eligibility. Using standardized coding forms, each selected paper was coded for study characteristics (study date, location, study design [RCT, cross-sectional, or non-RCT longitudinal studies], time frame used to measure adherence [previous day to past 2 weeks, past 2 weeks to 6 months, more than 6 months], recruitment setting, and method for adherence measurement [medication events monitoring system (MEMs), self-report or pharmacy records], participant characteristics [age, gender, race/ethnicity, percent who use/used alcohol and/or illicit drugs], treatment outcomes [clinical and/or immunological outcomes: HIV-1 viral load and CD4 count] and factors associated with better HIV-treatment response).

When more than one adherence measurement was used (e.g., MEMs and patient's self-report), data from all methods were collected, and an average adherence estimate was calculated, using information collected with the different methods. The adherence estimate included in our analysis referred to the percentage of patients rated as adherent, according to the cut-off and adherence measurement method adopted by each study. After potentially relevant studies were identified and data abstracted, the two investigators met to achieve consensus regarding eligibility.

\section{Statistical Analysis}

Standard meta-analytic methods were used (Cooper and Hedges 1994; Lipsey and Wilson 2001). A random-effects model for aggregating individual effect sizes was used, because it provides a more conservative estimate than a fixed-effects model of variance. This approach generates more accurate inferences since it recognizes studies as a sample of all potential studies and incorporates betweenstudy variability on the overall pooled estimation (Hedges and Vevea 1998; van Houwelingen et al. 2002). Thresholds for adherence were considered as greater than or equal to the cutoff levels used on each study, and the raw proportion of DUs recorded as optimally adherent by each study was used to pool the overall proportion, using the
DerSimonian-Laird random-effects method (Fleiss 1993; DerSimonian and Laird 1986).

The $I^{2}$ index was calculated as a measure of the overall variation in adherence proportion that was attributable to between-study heterogeneity (Higgins and Thompson 2002; Higgins et al. 2003). Higgins and Thompson (2002) proposed a tentative classification of $I^{2}$ values with the purpose of helping to interpret its magnitude. Thus, percentages of around $25 \%\left(I^{2} \leq 25\right), 50 \%\left(I^{2}=50\right)$, and $75 \%\left(I^{2} \geq 75\right)$ were interpreted as low, medium, and high heterogeneity, respectively. According to a recent review (Huedo-Medina et al. 2006), the $I^{2}$ index assesses not only heterogeneity in a meta-analysis but also the extent of that heterogeneity. It is considered a more appropriate procedure than the $Q$ test in assessing whether there is true heterogeneity among the studies in a meta-analysis (Higgins et al. 2003). Experts have demonstrated that the $I^{2}$ index exhibits higher power with a larger number of studies $(>20)$ with an average sample size higher than 80 individuals (Huedo-Medina et al. 2006).

We anticipated large between-study heterogeneity $\left(I^{2} \geq 75\right)$ considering the varied populations, the different definitions of 'optimal' adherence, and time frames used in the assessment of adherence. According to standard metaanalysis guidelines, when observational studies are pooled, heterogeneity of populations (e.g., US vs. international studies), design (e.g., case-control vs. cohort studies), and outcome (e.g., different studies yielding different relative risks that cannot be accounted for by sampling variation) is expected (Berlin 1995; Stroup et al. 2000).

Researchers have used a variety of time frames to capture adherence estimates, ranging from the previous day to previous year. The widely used AIDS Clinical Trials Group (ACTG) adherence instrument employs a 4-day time frame (Chesney et al. 2000). Shorter time frames have generally been considered to provide more accurate recall; however, a recent study suggested that 1 month time frame may be more accurate than 3- or 7-day periods (Lu et al. 2008). In an attempt to further contribute to this discussion, we aggregated studies according to their time frames into three subgroups: 1-14 days, 2 weeks to 6 months, and 12.5 years (no selected study used a time frame between 6 months and 1 year).

One forest plot was drawn for all 38 studies and additional figures were created according to those time frames. Forest plots illustrate the proportion of DUs recorded as optimally adherent by each study, its $95 \%$ confidence intervals (CI) and the overall DerSimonian-Laird pooled estimate.

A mixed-effects meta-regression model was used to specifically assess the underlying reasons for betweenstudy heterogeneity, whereby the proportion of DUs recorded as optimally adherent by each study was 
transformed to their logit value. The logit transformation (logistic function) allows for calculation because the values are approximately normally distributed in contrast with probability scores, which range from 0 to 1 (Viechtbauer 2007).

Results from univariate analyses with $P$-values $\leq 0.20$ were included in the multivariable analysis. The following covariates were included in the meta-regression multivariable model: time frame (according to the three categories indicated above), most frequently abused drug in the study sample (heroin, crack/cocaine, heroin and crack/cocaine, and abstinence), method of assessment for adherence (selfreport, pharmacy records, MEMS), and adherence cut-off $(>80 \%)$. Analyses were conducted using Stata version 10.0 (StataCorp, College Station, Tex) and R version 2.6.2 ( $\mathrm{R}$ Core Development Core Team 2004). Graphics were generated using StatsDirect version 2.5.2 (StatsDirect Ltd, Cheshire, England).

\section{Results}

\section{Study Characteristics}

Thirty-eight studies meeting inclusion/exclusion criteria were identified (Fig. 1). Approximately half assessed adherence using patient self-reports, while the remainder used a broad range of measurements (e.g., pharmacy

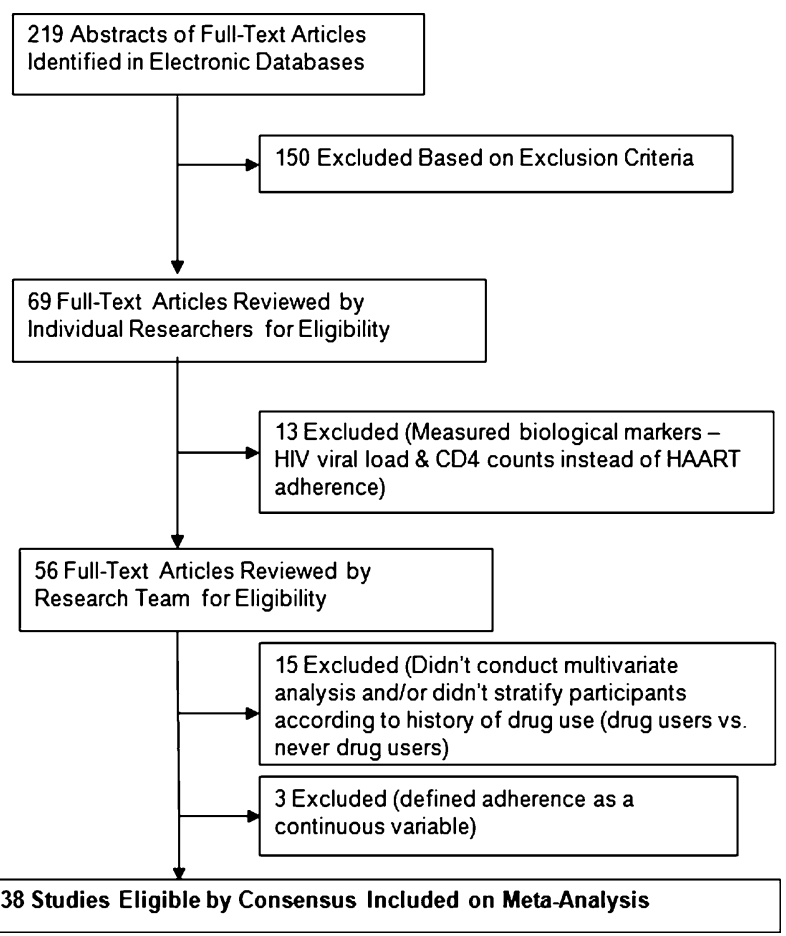

Fig. 1 Flow diagram of studies included in analysis records, MEMS, secondary data). Studies used heterogeneous cut-offs to define optimal adherence (range: 75$100 \%$ ). The 38 studies enrolled a total of 14,960 patients (range: 40-5,073; median 164). The 23 studies which used self-reported adherence enrolled a total of 5,150 patients (range: 42-636; median: 164), while 11 studies which evaluated adherence through pharmacy records and/or pill counts enrolled a total of 9,480 patients (range: $85-5,073$; median: 234). The four studies which evaluated adherence using MEMS or a combination of self-report and clinical data enrolled a total of 330 patients (range: 40-150; median: 70). We were unable to identify any studies meeting our eligibility criteria that were conducted in developing countries (Table 1).

Eleven studies defined optimal antiretroviral adherence as $100 \%$ uptake of the prescribed doses (Crisp et al. 2004; Haug et al. 2005; Martin et al. 2005; Palepu et al. 2003a, b, 2004b; Pradier et al. 2001; Purcell et al. 2004; Sharpe et al. 2004; Waldrop-Valverde and Valverde 2005; WaldropValverde et al. 2006). Eight studies assessed optimal adherence as greater than 95\% (Escobar et al. 2003; Kerr et al. 2004; Knowlton et al. 2006; Palepu et al. 2004b, 2006; Turner et al. 2003; Wood et al. 2003b, 2004b). Three studies used adherence greater than $90 \%$ (Arnsten et al. 2007; Gordillo et al. 1999; Hinkin et al. 2007); eight as greater than $80 \%$ (Altice et al. 2001; Avants et al. 2001; Bouhnik et al. 2002, 2005; Carrieri et al. 2003a; Clarke et al. 2003; Duran et al. 2001; Moatti et al. 2000); and one study as greater than $75 \%$ of prescribed medicines (Altice et al. 2004). One study used two different cut-offs-90 and $100 \%$ (Liu et al. 2006).

Six studies used a combination of adherence measurements, e.g., self-report and MEMS (Crystal et al. 2001; Kerr et al. 2005; Lucas et al. 2001; Martini et al. 2004; McNabb et al. 2001; Roca et al. 1999).

\section{Meta-Analysis}

The combined pooled adherence across 38 studies ( $N=14,960$ was 0.60 (95\% CI: $0.52-0.68$, Fig. 2 ), indicating that the pooled proportion of DUs recorded as optimally adherent by all studies was 0.60 . Due to the large between-study heterogeneity $\left(I^{2}=98.8 \%\right)$, we conducted subgroup analyses, re-calculating the pooled adherence according to their time frames: studies with a time frame $\leq 2$ weeks 17 studies $(N=3,795)$ between 2 weeks and 6 months 10 studies $(N=3,071)$ and studies with time frames between 1 year and 2.5 years $(11$ studies, $N=8,094)$. The pooled estimate for the shortest period was higher $\left(0.71 ; 95 \%\right.$ CI: $0.68-0.74, I^{2}=69.7 \%$; Fig. 3a) than the pooled estimated for the intermediate period $(0.54$, 95\% CI: $0.49-0.59, I^{2}=80.8 \%$, Fig. $\left.3 \mathrm{~b}\right)$. The group using a time frame greater than 1 year had the lowest pooled 
Table 1 Basic characteristics of selected studies, 1999-2007

\begin{tabular}{|c|c|c|c|c|}
\hline Source & $N$ & Population (\%) & Assessor & Threshold of measurement \\
\hline Arnsten et al. (2007) & 636 & $\operatorname{IDU}(100.0)^{\mathrm{a}}$ & Patient & $\geq 90 ;$ No. of pills taken/prescribed \\
\hline Hinkin et al. (2007) & 150 & Current IDU:102 (68.0) & MEMS $^{\mathrm{b}}$ & $\geq 90 ;$ No. of pills taken/prescribed \\
\hline Palepu et al. (2006) & 278 & IDU: 276 (99.3) & Pharmacy & $\begin{array}{l}\geq 95 \text {; No days patient receives HAART refills/No days } \\
\text { of follow-up }\end{array}$ \\
\hline $\begin{array}{l}\text { Waldrop-Valverde et al. } \\
\text { (2006) }\end{array}$ & 57 & IDU (100.0) & Patient & $=100 ;$ No. of pills taken/prescribed \\
\hline Liu et al. (2006) & 148 & $\begin{array}{l}\text { Cocaine: } 89(61.0) \\
\text { Heroin: } 39(27.0)\end{array}$ & Patient & $=100$ and $\geq 90 ;$ No. of pills taken/prescribed \\
\hline Knowlton et al. (2006) & 466 & $\begin{array}{l}\text { IDU }(100.0) ; 91 \% \\
\text { current } \mathrm{DU}^{\mathrm{c}}\end{array}$ & Patient & $\geq 95 ;$ No. of pills taken/prescribed \\
\hline $\begin{array}{l}\text { Waldrop-Valverde et al. } \\
\text { (2006) }\end{array}$ & 58 & IDU (100.0) & Patient & $=100 ;$ No. of pills taken/prescribed \\
\hline Haug et al. (2005) & 78 & 78 patients under $\mathrm{MMT}^{\mathrm{d}}$ & Patient/MEMS & $=100 ;$ No. of pills taken/prescribed \\
\hline Kerr et al. (2005) & 160 & IDUs & Patient/pharmacy & $\begin{aligned}= & 100 ; \text { HAART discontinuation: }(1) \text { picked up at least } \\
& \text { one prescription; (2) reported discontinuation of } \\
& \text { HAART for } \geq 1 \text { month }\end{aligned}$ \\
\hline Martin et al. (2005) & 100 & IDUs under MMT & Patient & $=100 ;$ No. of pills taken/prescribed \\
\hline Bouhnik et al. (2005) & 243 & IDUs & Patient & $\geq 80 \%$; No. of pills taken/prescribed \\
\hline Martini et al. (2004) & 214 & Ex-DU: 154 (71.9) & Patient & $\begin{array}{l}\text { No. error made (e.g., missing doses, interruption, } \\
\text { changing time); High adherence: } \leq 2 \text {; Medium: } 3-4 \text {; } \\
\text { Low: } \geq 5\end{array}$ \\
\hline Sharpe et al. (2004) & 784 & $\begin{array}{l}\text { Non DU: } 312(39.8) \\
\text { Crack users: } 173(22.1) \\
\text { Other drugs:299 (22.1) }\end{array}$ & Patient & $=100 ;$ No. of pills taken/prescribed \\
\hline Altice et al. (2004) & 62 & IDU & MEMS & $>75 \%$; No. of pills taken/prescribed \\
\hline Purcell et al. (2004) & 560 & IDU (100.0) & Patient & $=100 ; \%$ of those who missed at least one dose \\
\hline Palepu et al. (2004b) & 349 & IDU $(59 \%)$ & Patient & $\geq 95$; No. of pills taken/prescribed \\
\hline Palepu et al. $(2004 b)^{a}$ & 1,746 & $\begin{array}{l}\text { IDU/Ex-IDU: } 395 \\
\text { Never-IDU: } 1,351\end{array}$ & Pharmacy & $\begin{aligned}= & 100 ; \text { No days patient received HAART refills/No days } \\
& \text { of follow-up }\end{aligned}$ \\
\hline Crisp et al. (2004) & 137 & $\begin{array}{l}112(81.7 \%) \text { smoke } \\
\text { crack daily, last } 7 \text { days }\end{array}$ & Patient & $=100 ;$ No. of pills taken/prescribed \\
\hline Wood et al. (2004b) & 1,522 & $\begin{array}{l}\text { IDU:371 } \\
\text { Never-IDU: } 1,151\end{array}$ & Pharmacy & $\begin{array}{l}\geq 95 \text {; No days patient received HAART refills/No days } \\
\text { of follow-up }\end{array}$ \\
\hline Kerr et al. (2004) & 108 & IDU & Pharmacy & $\begin{array}{l}\geq 95 \text {; No days with filled prescription/No days under } \\
\text { therapy }\end{array}$ \\
\hline Wood et al. (2003b) & 1,422 & $\begin{array}{l}\text { IDU: } 359 \text { (25.3) } \\
\text { Never-IDU: } 1063 \text { (74.7) }\end{array}$ & Patient & $\begin{array}{l}\geq 95 \text {; Non adherents: Received HAART for less than } \\
95 \% \text { of the follow-up period }\end{array}$ \\
\hline Clarke et al. (2003) & 150 & IDU & Pharmacy & $\begin{array}{l}\geq 80 \text {; Pharmacy records demonstrate at least } 80 \% \text { rate of } \\
\text { HAART refills }\end{array}$ \\
\hline Turner et al. (2003) & 5,073 & 3,322 DU under treatment & Pharmacy & $\begin{array}{l}\geq 95 \text {; No days with filled prescription/No days under } \\
\text { therapy }\end{array}$ \\
\hline Palepu et al. (2003b) & 578 & $\begin{array}{l}\text { IDU: } 78 \text { (13) } \\
\text { Ex-IDU: } 96(17) \\
\text { Never IDU : } 404(70)\end{array}$ & Pharmacy & $\begin{aligned}= & 100 ; \text { No days patient received HAART refills/No days } \\
& \text { of follow-up }\end{aligned}$ \\
\hline Palepu et al. (2003a) & 234 & IDU: 128 (54.7) & Pharmacy & $\begin{aligned}= & 100 ; \text { No days patient received HAART refills/No days } \\
& \text { of follow-up }\end{aligned}$ \\
\hline Escobar et al. (2003) & 283 & $\begin{array}{l}\text { Alcohol and/or drug } \\
\text { use: } 203(71.7) ; \\
\text { IDUs: } 196(69.3)\end{array}$ & Pharmacy & $\geq 95 \%$; No. of pills taken/prescribed \\
\hline Carrieri et al. (2003a) & 96 & IDUs & Patient & $\geq 80 ;$ No. of pills taken/prescribed \\
\hline Bouhnik et al. (2002) & 210 & $\begin{array}{l}\text { Ex-IDU: } 114(54.3) \\
\text { IDU: } 96(45.7)\end{array}$ & Patient & $\geq 80 \%$; No. of pills taken/prescribed \\
\hline
\end{tabular}


Table 1 continued

\begin{tabular}{|c|c|c|c|c|}
\hline Source & $N$ & Population $(\%)$ & Assessor & Threshold of measurement \\
\hline Duran et al. (2001) & 57 & IDU (100.0) & Patient & $\geq 80 ;$ No. of pills taken/prescribed \\
\hline Lucas et al. (2001) & 558 & $\begin{array}{l}\text { Ex-IDU: } 376(49.2) \\
\text { IDU: } 199(26.0) \\
\text { NIDU: } 189(24.7)\end{array}$ & Patient & Non-adherence: $>2$ missed doses \\
\hline Altice et al. (2001) & 205 & $\begin{array}{l}\text { Incarcerated population } \\
\text { Ex-IDU (100.0) }\end{array}$ & Patient & $\geq 80$; No. of pills taken/prescribed \\
\hline McNabb et al. (2001) & 40 & IDU & $\begin{array}{l}\text { MEMS/ } \\
\text { Pill counts, } \\
\text { Patient }\end{array}$ & $\begin{array}{l}\text { MEMS: No. MEMS cap openings/No prescribed doses } \\
\text { Pill counts and Self report: No. of pills taken/prescribed }\end{array}$ \\
\hline Crystal et al. (2001) & 1,739 & IDU: $1,048(60.3 \%)$ & Pharmacy & $\begin{array}{l}\text { Proportion }(0-1.0) \text {; } \\
\text { No days on PI/NNRTI drugs/No days from first } \\
\text { prescription to the end of study }\end{array}$ \\
\hline Pradier et al. (2001) & 119 & IDU & Patient & $=100 \% ;$ No. of pills taken/prescribed \\
\hline Avants et al. (2001) & 42 & IDU & Patient & $\geq 80 ;$ No. of pills taken/prescribed \\
\hline Moatti et al. (2000) & 164 & Ex-IDU: 113 (68.9) & Patient & $\geq 80 ;$ No. of pills taken/prescribed \\
\hline Roca et al. (1999) & 133 & $\begin{array}{l}\text { IDU: } 95 \text { (71.0) } \\
\text { Non-IDU: } 38 \text { (29.0) }\end{array}$ & $\begin{array}{l}\text { Patient/Medical } \\
\text { Charts }\end{array}$ & $\begin{array}{l}\text { Adequate Adherence: (1) patients kept the } \\
\text { appointments; }(2)>80 \% \text { of prescribed doses; (3) } \\
\text { HIV-RNA level at least } 1.5 \log _{10} \text { below pre- } \\
\text { treatment level; Inadequate Adherence: (1) or (2) } \\
\text { were not met. Indeterminate Adherence: (1) and (2) } \\
\text { were met, but condition (3) was not }\end{array}$ \\
\hline Gordillo et al. (1999) & 366 & $\begin{array}{l}\text { Former IDUs: } 97(26.5) \\
\text { Current IDUs: } 65(17.8) \\
\text { Non-IDUs: } 204(55.7)\end{array}$ & Patient & $>90 \%$; No. of pills taken/prescribed \\
\hline
\end{tabular}

${ }^{a} I D U$ injection drug users

b MEMS medication event monitoring system

c $D U$ drug users

d MMT methadone maintenance therapy

adherence estimates (0.52; 95\% CI: $0.36-0.67, I^{2}=99.2 \%$, Fig. 3c).

A meta-regression model (Table 2) was fitted to evaluate major predictors of the between-studies heterogeneity, including the following covariates: time frame; substance used; type of adherence measurement, adherence cut-off and payment for receiving HAART. After adjustment, only the time frame remained associated with the between-study heterogeneity ( $P$-value $\leq 0.05)$. Compared to studies using the shorter time frame ( $\leq 2$ weeks), studies using a larger time frame had adherence estimates approximately 50\% lower. For studies using a time frame between 2 weeks and 6 months, the adjusted odds ratio (AOR) was 0.47 (95\% CI: 0.25-0.88), while for studies using a time frame higher than 1 year, AOR was 0.44 (95\% CI: 0.23-0.84).

\section{Treatment Outcomes}

Eighteen of the 38 studies evaluated HAART-related clinical outcomes (Avants et al. 2001; Bouhnik et al. 2005; Carrieri et al. 2003a; Duran et al. 2001; Knowlton et al.
2006; Lucas et al. 2001; McNabb et al. 2001; Moatti et al. 2000; Palepu et al. 2003a, b, 2004a, b, 2006; Pradier et al. 2001; Roca et al. 1999; Waldrop-Valverde et al. 2006; Wood et al. 2003a, 2004b) (Table 3).

Active drug use was identified by some authors as a barrier against better clinical outcomes (Lucas et al. 2001; Palepu et al. 2003a, b; Wood et al. 2004b). In these studies, active drug use (especially cocaine use) was identified as a stronger predictor of poor adherence and, in turn, failure to achieve/maintain better clinical outcomes over time. A study conducted in Baltimore, US, reported that active drug use was strongly associated with underutilization of HAART, non-adherence, and poorer responses to therapy; former drug users and non-drug users had comparable outcomes (Lucas et al. 2001).

Substitution therapy for opiate dependence was independently associated with HIV-1 RNA suppression according to several studies (Avants et al. 2001; Duran et al. 2001; Lucas et al. 2001; Moatti et al. 2000; Palepu et al. 2006). In a study by Palepu et al. (2006) in Vancouver, Canada, methadone maintenance therapy (MMT) 
Fig. 2 Pooled proportion of all HIV + DU adhering to antiretroviral therapy. Note: The combined data marker indicates the DerSimmonian-Laird combined proportion

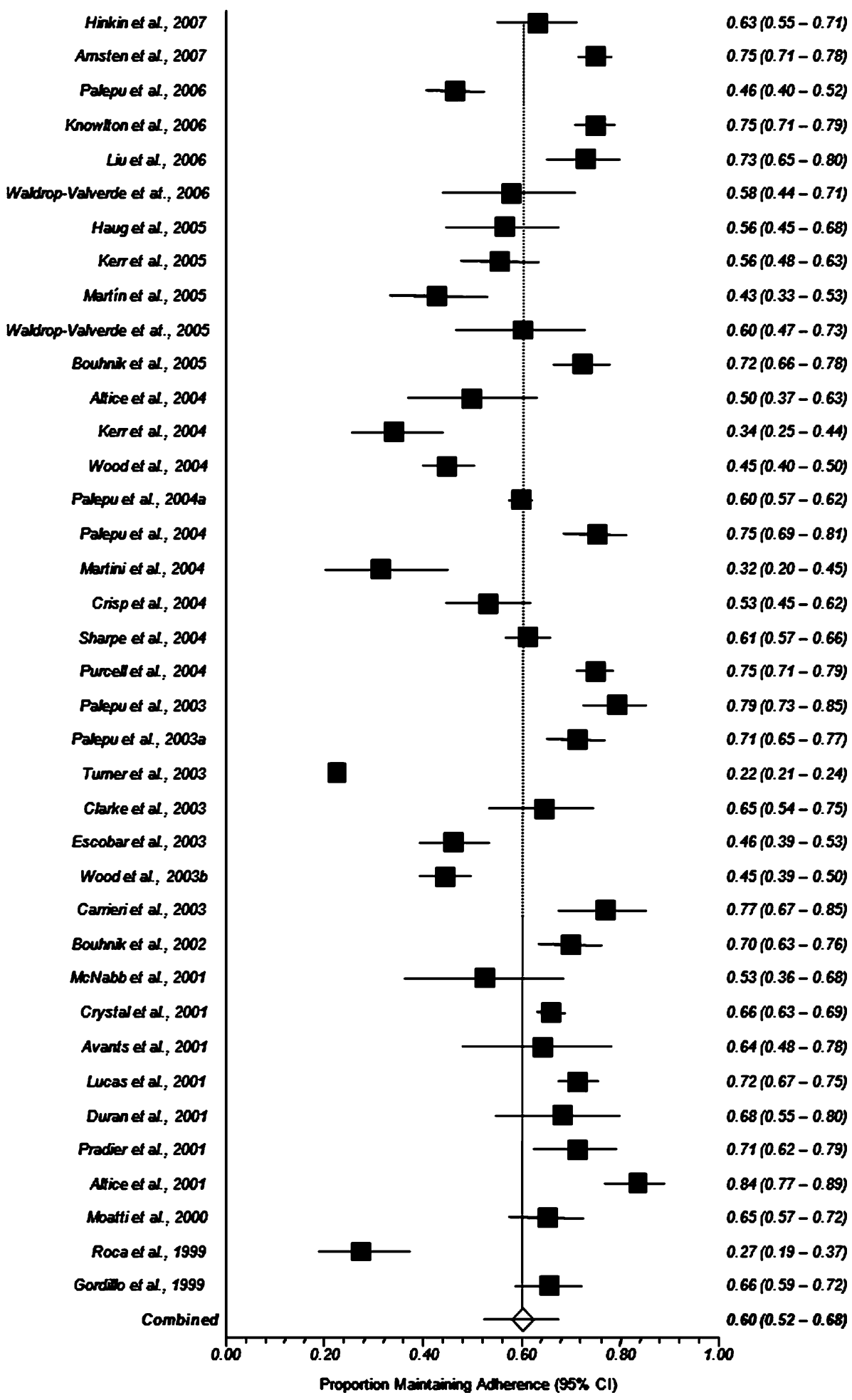

$0.63(0.55-0.71)$

$5(0.71-0.78)$

$0.75(0.74-0.79)$

$.73(0.65-0.80)$

$(0.44-0.71)$

$0.56(0.45-0.68)$

$0.60(0.47-0.73)$

$0.72(0.66-0.78)$

$0.34(0.25-0.44)$

$0.450 .40-0.50)$

$0.00 .57-0.62)$

$0.32(0.20-0.45)$

0.62

$0.75(0.71-0.79)$

$0.79(0.73-0.85)$

$10.65-0.77)$

$22(0.21-0.24)$

$0.65(0.54-0.75)$

$0.46(0.39-0.53)$

$0.45(0.39-0.50)$

$0.67-0.85)$

$0.53(0.36-0.68)$

$0.66(0.63-0.69)$

$0.64(0.48-0.78)$

$0.72(0.67-0.75$

$0.68(0.55-0.80)$

$0.71(0.62-0.79)$

$0.27(0.19-0.37)$

$0.66(0.59-0.72)$

$0.60(0.52-0.68)$ was associated with both HIV-1 RNA suppression (AOR:1.34; 95\% CI:1.00-1.79) and CD4 cell count increase (AOR: 1.58; 95\% CI:1.26-1.99).

Psychiatric conditions, particularly depression, were found to be associated with poorer response to HAART. Bouhnik et al. (2005) found that besides non-adherence, having a higher level of depressive symptoms following HAART initiation was highly associated with HIV clinical progression (HR: 5.3; 95\% CI: 2.2-13.0; $P<10^{-3}$ ).

In some studies, indicators of low socioeconomic status were also associated with poorer clinical and virologic response among HIV-positive DU receiving HAART. 

all HIV + DU adhering to ART, studies using 2 weeks or less for adherence recall. b Pooled proportion of all $\mathrm{HIV}+\mathrm{DU}$ adhering to ART, studies using 16 days to 6 months for adherence recall. c Pooled proportion of all HIV + DU adhering to ART, studies using more than 6 months of adherence recall. Note: The combined data marker indicates the

DerSimmonian-Laird combined proportion
Fig. 3 a Pooled proportion of

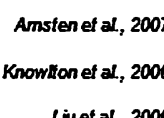

Livef al, 2006 Waldrop-Valverde ef af., 2006 Waldrop-Valverde ef af., 2005 Bounnik ef al, 2005 Altice ef al, 2004 Purcellef at, 2004 Camierief at, 2003 Bounnik ef at., 2002 Avants ef al, 2001 Lucas ef al, 2001 Pradier ef at, 2001 Altice ef at, 2001 Mbattiet al, 2000 Gordiulo ef al, 1999 Combined 0.00
A

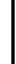

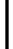
Duran ef al, 2001
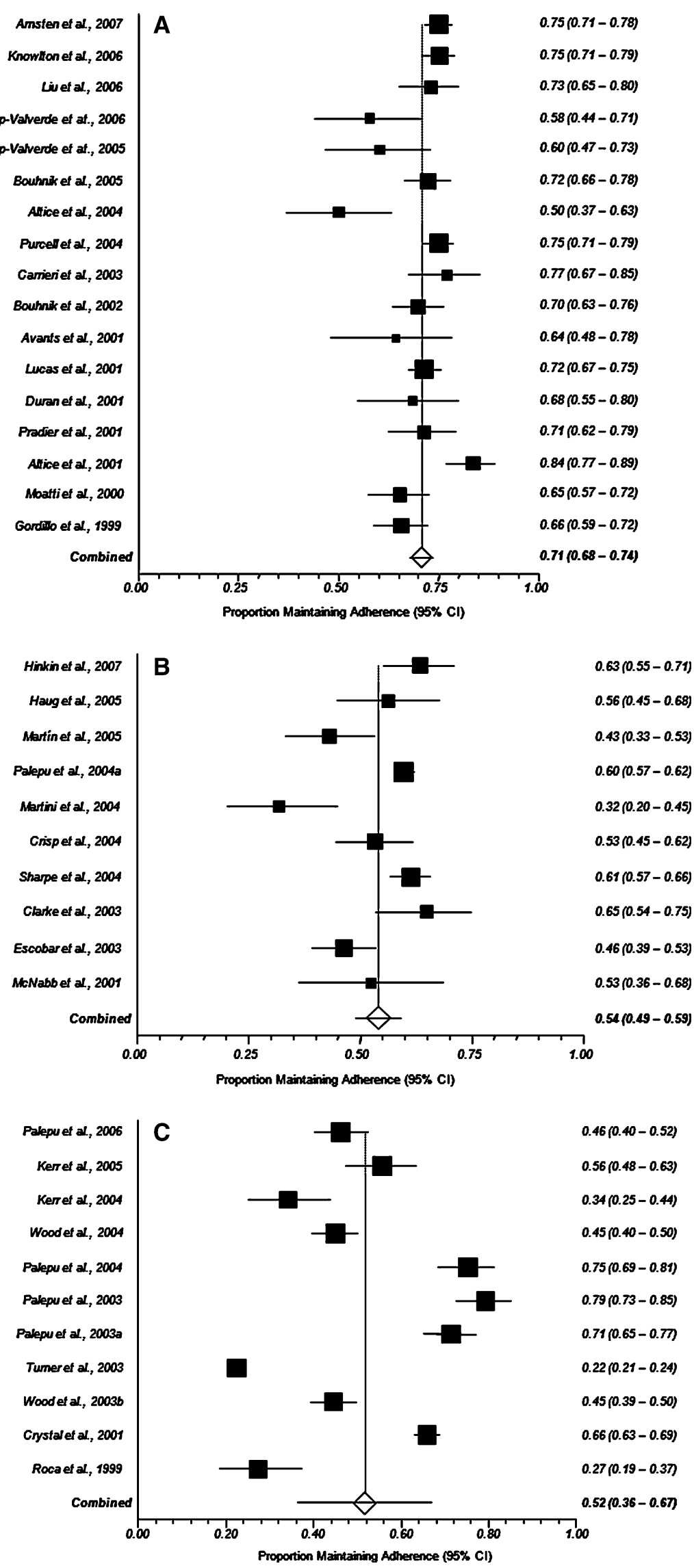
Table 2 Covariates associated with "between-studies" heterogeneity according to multivariable logistic regression

\begin{tabular}{lll}
\hline Variable & OR $(95 \% \mathrm{CI})$ & AOR $(95 \% \mathrm{CI})$ \\
\hline $\begin{array}{l}\text { Time frame } \\
\quad \text { Last day-15 days }\end{array}$ & 1.00 & 1.00 \\
$\quad$ 16 days-6 months & $0.49(0.32-0.77)$ & $0.47(0.25-0.88)^{*}$ \\
$\quad$ 1-2.5 years ${ }^{\mathrm{a}}$ & $0.46(0.30-0.71)$ & $0.44(0.23-0.84)^{*}$ \\
Drug used & & \\
$\quad$ Heroin and cocaine & 1.00 & 1.00 \\
$\quad$ Heroin & $1.33(0.74-2.38)$ & $0.90(0.50-1.63)$ \\
$\quad$ Crack/cocaine & $0.94(0.37-2.38)$ & $1.21(0.44-3.36)$ \\
$\quad$ Abstinence & $3.55(0.54-13.38)$ & $2.12(0.62-7.25)$ \\
Measurement & & \\
$\quad$ Self report & 1.00 & 1.00 \\
$\quad$ Pharmacy records & $0.64(0.40-1.01)$ & $1.12(0.60-2.11)$ \\
$\quad$ MEMS-cap & $0.69(0.34-1.40)$ & $1.13(0.51-2.48)$ \\
Adherence cut-off $(>80 \%)$ & $1.62(0.90-2.90)$ & $1.46(0.83-2.58)$ \\
Adherence cut-off $(>90 \%)$ & $0.86(0.55-1.34)$ & - \\
Adherence cut-off $(>95 \%)$ & $0.73(0.48-1.11)$ & - \\
Adherence cut-off $(=100 \%)$ & $1.14(0.72-1.81)$ & - \\
Paying for treatment & $1.15(0.75-1.78)$ & - \\
\hline
\end{tabular}

$C I$ confidence interval; $O R$ odds ratio; $A O R$ adjusted odds ratio (adjusted for all variables listed in the table); MEMS medication events monitoring system

${ }^{a}$ No selected study used a time frame between 6 months and 1 year * $P$-value $\leq 0.05$

These studies identified access to social support and stable housing as key aspects to achieve/maintain both HAART adherence and viral suppression (Avants et al. 2001; Carrieri et al. 2003a; Knowlton et al. 2006; Lucas et al. 2001; Palepu et al. 2003a, b, 2004a, b, 2006; Wood et al. 2003b).

Characteristics directly related to the class of antiretroviral drugs (two nucleosides + protease inhibitor vs. nonnucleoside reverse transcriptase inhibitors), time on HAART (more experienced vs. less experienced patients), and levels of viral load/CD4 cells counts at the time of HAART initiation were also identified as cofactors independently associated with viral suppression and immune reconstitution (Knowlton et al. 2006; Palepu et al. 2003a, b, 2004a, 2006).

\section{Discussion}

Findings from this meta-analysis suggest that adherence to HAART among HIV-positive drug users falls within the range observed among PLWHA in general, which is approximately $60 \%$. However, the strong heterogeneity among different studies jeopardizes efforts to standardize the assessment of adherence and to derive evidence-based guidelines about how adherence should be measured and how existing measures can be improved.

A recent meta-analysis evaluating 31 studies measuring adherence to HAART among adult PLWHA from North America $(N=17,573)$ reported a pooled estimate of $55 \%$ (95\% CI: 49-62\%), slightly lower than that identified by our study, but with overlapping confidence intervals (Mills et al. 2006). Non-adherence to HAART in adult populations has ranged from 33 to $88 \%$, depending on the methods by which adherence is defined and evaluated (Friedland and Williams 1999).

A recent paper by Bangsberg (2006) found that viral suppression and better clinical outcomes was common among patients with a $54-100 \%$ mean adherence level, if the patient was using non-nucleoside reverse-transcriptaseinhibitor regimens instead of unboosted protease inhibitor regimens which are known to be partially suppressive. Although perfect adherence is an important goal, viral suppression and better clinical outcomes are possible with moderate adherence to potent regimens, such as those levels identified by our study. Our systematic review (Malta et al. 2008) and meta-analysis favor the conclusion that drug users may achieve good adherence levels and clinical outcomes if given access to HAART; outcomes are improved when HAART is offered in the context of social supports and methadone maintenance treatment (Clarke et al. 2003; Malta et al. 2008; Moatti et al. 2000; Palepu et al. 2006).

Evidence-based studies on barriers and facilitators to adherence among HIV-positive DU have been scarce, particularly in developing countries. This is of concern given that the largest HIV epidemics among DU are now taking place in developing/transitional countries, such as eastern Europe, as well as south east, south and central Asia (Mathers et al. 2008). Over the coming decades, PLWHA from developing countries will constitute a growing proportion of the World's HAART recipients as treatment roll-out progresses. Studies on HAART utilization and adherence in low and middle income countries are urgently needed in order to inform efforts to implement and expand HAART use in regions where it is needed the most.

Our meta-analysis found that studies using shorter time frames yielded more homogeneous estimates of adherence among HIV-positive DUs. A recent report comparing selfreported and MEMS adherence among HIV-positive patients found less overestimation of self-reported adherence with a 1 month time frame than with 3- or 7-day time frames among participants with higher socioeconomic status-94\% had finished high school ( $\mathrm{Lu}$ et al. 2008). There is no consensus about the more accurate measurement of HAART adherence among HIV-positive patients (Chesney 2006; Pearson et al. 2007a, b; Simoni et al. 2007). Clinical guidelines for the management of HIV- 
Table 3 Treatment outcomes for 18 studies with available data, 1999-2006

\begin{tabular}{|c|c|}
\hline Source & Major treatment outcomes \\
\hline Palepu et al. (2006) & $\begin{array}{l}\text { Factors associated with HIV-1 RNA suppression: } \\
\left.\text { MMT }^{\mathrm{a}} \text { (AOR }^{\mathrm{b}} 1.34 ; 95 \% \text { CI } 1.00-1.79\right) \\
\geq 95 \% \text { HAART }^{\mathrm{c}} \text { adherence (AOR2.86; 95\% CI 2.19-3.75) } \\
\text { Older age, per } 10 \text { years (AOR1.47; 95\% CI 1.13-1.91) } \\
\text { Time on HAART (AOR } 1.08 ; 95 \% \text { CI 1.02-1.14) } \\
\text { Factors associated with CD4 cell count rise: } \\
\text { MMT (AOR } 1.58 ; 95 \% \text { CI } 1.26-1.99) \\
\geq 95 \% \text { HAART adherence (AOR } 1.42 ; 95 \% \text { CI } 1.13-1.78) \\
\text { Baseline CD4 cell count (AOR } 1.27 ; 95 \% \text { CI } 1.18-1.36)\end{array}$ \\
\hline $\begin{array}{l}\text { Waldrop-Valverde et al. } \\
\text { (2006) }\end{array}$ & $\begin{array}{l}\text { IDU }^{\mathrm{d}} \geq 95 \% \text { adherents to HAART versus IDU }<95 \% \text { adherents to HAART (previous day adherence): } \\
\text { Lower mean HIV RNA log-transformed copies }(\mathrm{ml}): 8.7( \pm 2.5) \text { versus } 10.0( \pm 2.16) ; P=0.047 \\
\text { No differences in mean CD4 log-transformed cell counts }\left(\mathrm{mm}^{3}\right): 5.3( \pm 1.2) \text { versus } 5.2( \pm 1.2) ; P=0.839\end{array}$ \\
\hline Knowlton et al. (2006) & $\begin{array}{l}239 / 350(68.3 \%) \text { adherents patients had detectable HIV-1 viral load }(P<0.05) \\
\text { Factors associated with HIV-1 RNA suppression: } \\
\text { CD4 count }>200 \text { : AOR: } 2.75,95 \% \text { CI: } 1.63-4.63(P<0.001) \\
\text { High social support: AOR: } 4.86,95 \% \text { CI: } 1.08-21.93(P<0.05) \\
\text { Better patient } \times \text { provider communication: AOR: } 1.57,95 \% \text { CI: } 1.01-2.45(P<0.05) \\
\text { Stable housing: AOR: } 3.67,95 \% \text { CI: } 1.04-13.04(P<0.05)\end{array}$ \\
\hline Bouhnik et al. (2005) & $\begin{array}{l}\text { HIV clinical progression faster for non-adherents at baseline: adherents versus non-adherents (log-rank test, } \\
\quad P=0.02) \\
\text { HIV clinical progression faster for non-adherents during follow-up: adherents versus non-adherents (Cox model, } \\
\quad P<10^{-3} \text { ) } \\
\text { HIV clinical progression faster for depressive patients at baseline: adherents versus non-adherents (log-rank test, } \\
\quad P<10^{-3} \text { ) } \\
\text { HIV clinical progression faster for depressive patients during follow-up: adherents versus non-adherents (Cox model, } \\
\quad P<10^{-3} \text { ) }\end{array}$ \\
\hline Palepu et al. (2003b) & $\begin{array}{l}\text { Factors negatively associated with HIV-1 RNA suppression: } \\
\text { History of incarceration within } 12 \text { months of initiating HAART: } \text { HHR }^{\mathrm{e}}: 0.68 ; 95 \% \text { CI: } 0.51-0.89 \text {; } \\
\text { History of drug injection: AHR: } 0.79 ; 95 \% \text { CI: } 0.69-0.91 \text {; } \\
\text { Two nucleosides + PI versus two nucleosides + NNRTI (AHR: } 0.77 ; 95 \% \text { CI: } 0.69-0.87 ; \\
\text { Higher baseline HIV-1 RNA: AHR: } 0.66 ; 95 \% \text { CI: } 0.62-0.70 \\
\text { Factors positively associated with HIV-1 RNA suppression: } \\
\text { Higher adherence to HAART: AHR: } 1.38 ; 95 \% \text { CI: } 1.34-1.42 \\
\text { Among participants incarcerated in the first year of starting HAART, the time spent in jail was positively associated } \\
\text { with HIV-1 RNA suppression: HR: } 1.06 ; 95 \% \text { CI: } 1.02-1.10\end{array}$ \\
\hline Palepu et al. (2004a) & $\begin{array}{l}\text { Achieved HIV-1 RNA suppression: } 1,318(76.9 \%) \\
\text { Factors associated HIV-1 RNA suppression: } \\
\text { No history of incarceration: } 96 \% \text { versus } 89 \%(P<0.001) \\
\text { Older age: } 37 \text { versus } 36 \text { years }(P<0.001) \\
\text { Higher median adherence: } 100 \% \text { versus } 58 \%(P<0.001) \\
\text { Higher physician HIV-related experience: } 59 \text { versus } 38(P=0.002) \\
\text { Male gender }(P<0.001) \\
\text { Never IDU }(P<0.001)\end{array}$ \\
\hline Wood et al. (2004b) & $\begin{array}{l}\text { CD4 cell count response (probability of first CD4 cell count gain of : } \geq \text { cells } / \mathrm{mm}^{3} \text { from baseline) } \\
\text { Patients with history of injection drug use had lower CD4 response rates }(\log \text {-rank: } P<0.05 \text { ) } \\
\text { Among patients with adherence } \geq 95 \% \text {, no statistical difference was found between IDU and Never-IDU (log-rank: } \\
\quad P=0.349 \text { ) }\end{array}$ \\
\hline
\end{tabular}


Table 3 continued

\begin{tabular}{|c|c|}
\hline Source & Major treatment outcomes \\
\hline Wood et al. (2003a) & $\begin{array}{l}\text { Cumulative suppression of HIV viral load among all participants, after } 12 \text { months of HAART: } \\
\text { Non-IDU versus IDU: } 70.8 \% \text { versus } 51.4 \% \text { (log-rank: } P<0.001 \text { ) } \\
\text { Cumulative suppression of HIV viral load among participants } \geq 95 \% \text { adherents, after } 12 \text { months of HAART: } \\
\text { Similar between non-IDU versus IDU: log-rank, } P=0.12 \\
\text { HIV viral load suppression in multivariate model (adjusted for adherence, sex, age, PI use, baseline CD4 and HIV } \\
\text { viral load and date of therapy initiation): } \\
\text { Similar between non-IDU versus IDU: Adjusted RH: } 0.9,95 \% \text { CI } 0.7-1.0 \\
\text { Cumulative HIV RNA rebound rate, among all participants who achieved viral suppression: } \\
\text { Non-IDU versus IDU: } 23.8 \% \text { versus } 34.7 \text { (log-rank, } P<0.001 \text { ) } \\
\text { Cumulative HIV RNA rebound rate, among participants } \geq 95 \% \text { adherents who achieved viral suppression: } \\
\text { Similar between non-IDU and IDU: log-rank, } P=0.12 \\
\text { Rates of HIV rebound in multivariate model (adjusted for adherence, sex, age, PI use, baseline CD4 and HIV viral } \\
\text { load and date of therapy initiation): } \\
\text { Similar between non-IDU versus IDU: Adjusted RH: } 1.3,95 \% \text { CI } 1.0-1.6\end{array}$ \\
\hline Palepu et al. (2003a, b) & $\begin{array}{l}\text { Factors associated with HIV-1 RNA suppression, former and non-IDU: } \\
\text { Adherence to HAART, per } 10 \% \text { : AOR: } 1.33,95 \% \text { CI: } 1.14-1.55 \text {; } \\
\text { Lower baseline HIV-1 RNA: AOR: } 2.16,95 \% \text { CI: } 1.18-4.18 \text {; } \\
\text { Two nucleosides plus NNRTI versus two nucleosides plus PI: AOR: } 4.67,95 \% \text { CI: } 1.55-14.1 \text {; } \\
\text { Months on therapy: AOR: } 1.11,95 \% \text { CI: } 1.07-1.15 \\
\text { Factors associated with HIV-1 RNA suppression, active drug users: } \\
\text { Lower baseline HIV-1 RNA: AOR: } 4.85,95 \% \text { CI: } 1.34-17.5 \text {; } \\
\text { Two nucleosides plus NNRTI versus two nucleosides plus PI: AOR: } 7.19,95 \% \text { CI: } 1.46-35.7 \text {; } \\
\text { Months on therapy: AOR: } 1.13,95 \% \text { CI: } 1.06-1.20\end{array}$ \\
\hline Palepu et al. (2003a) & 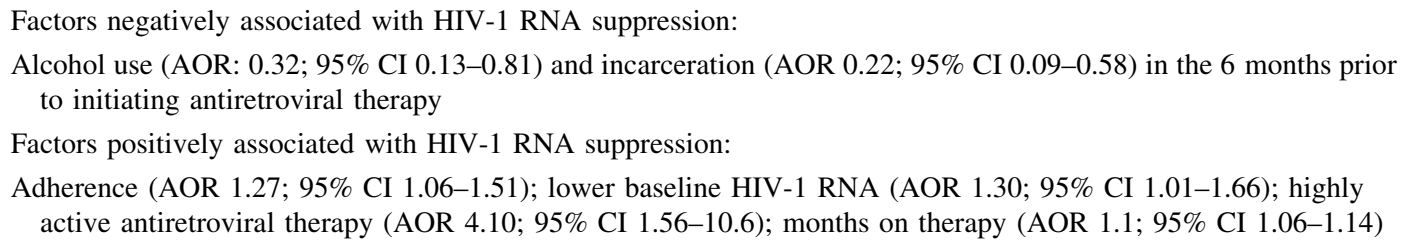 \\
\hline Carrieri et al. (2003a) & $\begin{array}{l}\text { Undetectable HIV viral load, IDU adherents versus IDU non-adherents }(<500 \text { copies } / \mathrm{ml}) \text { : } \\
56.8 \% \text { versus } 36.3 \%(P=0.09)\end{array}$ \\
\hline Duran et al. (2001) & $\begin{array}{l}\text { Median viral load, last visit: } \\
\text { Non-adherent } 3.80 \log _{10} \text { copies/ml, IQR: } 2.60-5.04 \\
\text { Adherents patients: } 2.30 \log _{10} \text { copies/ml, IQR: } 2.30-3.64 \\
\text { Strictly adherent: } 2.30 \log _{10} \text { copies/ml, IQR: } 2.30-3.45 \text {, Mann-Whitney test }(P=0.001) \\
\text { Proportion of patients with undetectable viral load, last visit: } \\
\text { Non-adherent }(22.2 \%) \text {, versus adherent }(69.2 \%) \text { versus 'strictly adherent' }(73.1 \%) ; \chi^{2} \text { test }(P=0.002) \\
\text { Median CD4 cell counts, last visit: } \\
\text { Non-adherent: } 403 \text { cells } / \mathrm{mm}^{3}, \mathrm{IQR}: 218-534 \\
\text { Adherents: } 446 \text { cells } / \mathrm{mm}^{3}, \mathrm{IQR}: 355-517 \\
\text { Strictly adherents: } 518 \text { cells } / \mathrm{mm}^{3}, \mathrm{IQR}: 389-655 \text { Mann-Whitney test }(P=0.06)\end{array}$ \\
\hline Lucas et al. (2001) & $\begin{array}{l}\text { Active drug use status was correlated with lower virologic and immunologic response to HAART } \\
\text { Median HIV-1 RNA reduction: } \\
\text { Active drug users } 0.8 \log _{10} \text { copies/ml versus } 1.7 \text { in nonusers and } 1.6 \text { in former users }(P<0.001 \text {, both comparisons) } \\
\text { Median CD } 4 \pm \text { lymphocyte count increase: } \\
\text { Active drug users } 65 \text { cells } / \mathrm{mm}^{3} \text { versus } 116 \text { in nonusers and } 122 \text { in former users ( } P=0.003 \text { both comparisons) } \\
\text { Prevalence of patients achieving undetectable HIV viral load ( } \leq 400 \text { copies } / \mathrm{ml}) \text { : } \\
32 \% \text { of active drug users versus } 46 \% \text { of nonusers versus } 44 \% \text { of former users }(P=0.02 \text { both comparisons) }\end{array}$ \\
\hline
\end{tabular}


Table 3 continued

\begin{tabular}{|c|c|}
\hline Source & Major treatment outcomes \\
\hline McNabb et al. (2001) & $\begin{array}{l}\text { After } 3 \text { months of follow-up, MEMs cap adherence was associated with virologic success }(P=0.0231) \text {; } \\
\text { Subjects with stable, undetectable or decreasing virus load had better rates of adherence }(70 \%-80 \%) \text { than those } \\
\text { subjects with stable virus load }>400 \text { copies/ml or an increasing virus load }(27 \%-51 \%)\end{array}$ \\
\hline Pradier et al. (2001) & $\begin{array}{l}\text { PI Adherence and Plasma HIV-1 RNA viral load: } \\
\text { G1—participants w/detectable viral load and decreased } \leq 0.5 \log \text { of plasma HIV viral load: } 17(56.7 \%) \text { were } 100 \% \\
\text { adherents } \\
\text { G2_participants w/detectable viral load and decreased }>0.5 \log \text { of plasma HIV viral load: } 22(64.7 \%) \text { were } 100 \% \\
\text { adherents } \\
\text { G3—participants w/undetectable viral load and decreased }<2.3 \log \text { of plasma HIV viral load: } 46 \text { (83.6\%) were } 100 \% \\
\text { adherents } \\
(P=0.02 \text {, G1 vs. G2 vs. G3) } \\
\text { Mean increase in CD4 } \pm \text { count/mm3: } \\
\text { G1-participants w/detectable viral load and decreased } \leq 0.5 \log \text { of plasma HIV viral load: } 3.2 \pm 106 \\
\text { G2-participants w/detectable viral load and decreased }>0.5 \log \text { of plasma HIV viral load: } 143 \pm 147 \\
\text { G3-participants w/undetectable viral load and decreased }<2.3 \log \text { of plasma HIV viral load: } 123 \pm 160 \\
(P=0.0002, \mathrm{G} 1 \text { vs. G2 vs. G3) }\end{array}$ \\
\hline Avants et al. (2001) & $\begin{array}{l}\text { Ratio of missed prescriptions decreased during methadone stabilization: } \\
\text { Week 1: } 0.24( \pm 0.32) \\
\text { Week 2: } 0.13( \pm 0.24) \\
\text { Week 3: } 0.11( \pm 0.22) \\
\text { Week 4: } 0.13( \pm 0.25)\end{array}$ \\
\hline Moatti et al. (2000) & $\begin{array}{l}\text { Median viral load, non-adherent versus adherents patients: } 3.9 \log _{10} \text { copies } / \mathrm{ml} \text { versus } 2.7 \log _{10} \text { copies } / \mathrm{ml} \\
\quad(P=0.008) \\
\text { Median decrease in viral load before and after HAART initiation, non-adherent versus adherents patients: } \\
-0.53 \log _{10} \text { copies } / \mathrm{ml} \text { versus }-1.04 \log _{10} \text { copies } / \mathrm{ml}(P=0.025) \\
\text { Percentage of patients with undetectable viral load or with a viral load decrease higher than } 1 \log _{10} \text {, non-adherent } \\
\quad \text { versus adherents patients: } \\
40.3 \% \text { versus } 57.0 \%(P=0.04) \\
\text { Median increase of CD } 4 \text { cell counts among non-adherent versus adherents patients: } \\
46 \times 10^{6} / 1 \text { versus } 80 \times 10^{6} / 1(P=0.82)\end{array}$ \\
\hline Roca et al. (1999) & $\begin{array}{l}\text { Virological efficacy was reached in }>40 \% \text { of patients of both groups in all visits (IDU and non-IDUS) } \\
\text { After } 6 \text { months of treatment: } \\
35(43 \%) \text { participants presented a CD } 4 \text { cell count increase }>100 \times 10^{6} / 1 \\
47(58 \%) \text { participants achieved undetectable HIV RNA ( } \leq 200 \text { copies/ml) } \\
\text { CD4 cell count and HIV RNA responses were similar in both groups. } \\
\text { No differences were observed in immunological, virological or clinical efficacy between IDU and non-IDU }\end{array}$ \\
\hline
\end{tabular}

${ }^{\text {a }} M M T$ methadone maintenance therapy

b $A O R$ adjusted odds ratio

c $H A A R T$ highly active antiretroviral therapy

d $I D U$ injection drug users

positive drug users favor the use of shorter time frames to evaluate their adherence to HAART, following, for instance, the widely used ACTG adherence instrument that employs a 4-day time frame (Chesney et al. 2000; New York State Department of Health 2005). However, it was not possible to disentangle the specific influence of different time frames and the underlying conceptual strategies to assess adherence used by clinical trial groups.
Measuring long-term adherence is particularly challenging, since adherence often fluctuates (Carrieri et al. 2003b) and may decrease over time (Howard et al. 2002; Parruti et al. 2006; Pearson et al. 2007a, b; Pinheiro et al. 2002). According to a study conducted by the HIV Epidemiology Research Study (HERS) with HIV-positive drug users, participants' adherence declined from $64 \%$ at 1 month to $45 \%$ at 6 months after initiation of HAART 
(Arnsten et al. 2001). Nevertheless, Parruti et al. (2006) found that adherence remained fairly stable up to 24 months of follow-up, declining about 5\% every 6 months thereafter. Therefore, the differences observed in our analyses relating to short versus long-term adherence may represent a true decrease in adherence over time. Further studies are needed to clarify this issue.

Most studies reporting higher adherence were conducted among DU receiving HAART in structured settings, particularly those offering both addiction treatment and psychosocial support (Purcell et al. 2004) and/or directly observed therapy (Altice et al. 2004; Smith-Rohrberg et al. 2006). In particular, DU engaged in opioid substitution therapy significantly reduced their drug using habits, have better adherence to HAART, and usually achieved the expected HIV-1 RNA suppression and CD4 cell count response to HAART (Clarke et al. 2003; Moatti et al. 2000; Palepu et al. 2006). These findings suggest that the extent to which one's daily life is routinized and stabilized may improve adherence and, as a consequence, responses to HIV treatment among DU.

Although opioid substitution therapy may be associated with better HAART adherence among people who use heroin and other opioids, illicit stimulant use remains a key problem. HIV-positive active cocaine users may seek treatment with considerable delay, initiating HAART in more advanced disease stages (Cofrancesco et al. 2008; Martín-Sánchez et al. 2002), which, in turn, decreases its effectiveness. Methamphetamine use may also represent similar challenges (Hinkin et al. 2007) but no studies of HAART adherence among exclusively methamphetamine users met our study's eligibility criteria. Active alcohol abuse (Palepu et al. 2003a, b) is also associated with lower adherence to HAART. Patients co-infected with HCV and other STIs may also have worse clinical outcomes (Miller et al. 2005; Vissoci Reiche et al. 2008).

We identified a high degree of between-study heterogeneity in adherence estimates, since selected studies used a wide range of cut-offs to define optimal adherence (range: 75-100\%), assessed adherence through different measurements, used different time frames, and collected data in a variety of research centers and clinical settings. Due to this high degree of heterogeneity, which was not completely explained either by sub-groups analysis or by meta-regression, our pooled results need to be viewed with caution. The exclusion criteria for our analysis may have limited the generalizability of our findings. A recent metaanalysis evaluating HAART adherence among PLWHA comprising a majority of observational studies and a few randomized controlled trials revealed a high heterogeneity with an $I^{2}$ of $98.4 \%$ (Mills et al. 2006). Heterogeneity between studies is a key issue for further studies and should be the object of concerted efforts toward standardization. Multicenter studies and especially cross-cultural studies are sorely needed.

This meta-analysis has other limitations. We aimed to reduce reviewer bias by conducting abstraction independently, in parallel. We cannot, however, know to what extent reporting bias may have influenced our conclusions. Reporting bias, particularly in studies relying only on selfreport may have limited our ability to accurately identify the actual levels of adherence to HAART among HIV-positive DU. The accuracy of such reports cannot be verified in the context of the current study. However, in the context of our analysis, differential biases were not made evident by meta-regression.

We were unable to identify studies conducted in developing countries, making it impossible to generalize our findings to those settings. Our review relies on information reported in peer-reviewed scientific publications of studies conducted in developed countries, which did not represent those DU living in specific contexts, such as eastern Europe, where the AIDS epidemic is mainly driven by drug using population and access to HAART is uneven (WHO/UNAIDS 2007).

While there is significant reluctance among medical care providers to deliver HAART to DU, the evidence supporting this decision is limited (Aceijas et al. 2006; Malta et al. 2008; Vlahov and Celentano 2006). Overcoming stigma and discrimination towards HIV-positive DU, and improving the quality and efficacy of HIV care and management is necessary to achieve optimal clinical outcomes for this marginalized population. Much has to be done in terms of a comprehensive monitoring of adherence, including the standardization of methods, time frames and the very covariates to be explored as predictors and/or confounders. Our meta-analysis suggests that HIV-infected DUs can achieve comparable adherence levels to non-drug using populations, and argues strongly against discriminatory polices that prevent DUs from accessing HAART.

Acknowledgments Sincere thanks are due to the technical assistance of Dr. Edward Mills, from Toronto, Canada. Dr. Mills is a researcher and professor from the Centre for International Health and Human Rights Studies and from the Oxford University, who kindly guided us during our analysis using the StatsDirect software. Sincere thanks are also due to Dr. Chuck Cleland, statistician and researcher from the Institute for AIDS Research, National Development and Research Institutes from New York; and to Dr. Wolfgang Viechtbauer, professor from the Department of Methodology and Statistics, University of Maastricht, from the Netherlands. Both Dr. Cleland and Dr. Viechtbauer kindly answered to our doubts while using the R-software. Dr. Malta acknowledges support from the National Institute on Drug Abuse and the Inter-American Drug Abuse Control Commission (NIDA/CICAD Research Award). Dr. Strathdee acknowledges support from the Fogarty International Center, grant R25-TW007500. 


\section{References}

Aceijas, C., Oppenheimer, E., Stimson, G. V., Ashcroft, R. E., Matic, S., \& Hickman, M. (2006). Antiretroviral treatment for injecting DU in developing and transitional countries 1 year before the end of the "Treating 3 million by 2005. Making it happen. The WHO strategy" ("3 by 5"). Addiction (Abingdon, England), 101, 1246-1253. doi:10.1111/j.1360-0443.2006.01509.x.

Altice, F. L., Mezger, J. A., Hodges, J., Bruce, R. D., Marinovich, A., Walton, M., et al. (2004). Developing a directly administered antiretroviral therapy intervention for HIV-infected DU: Implications for program replication. Clinical Infectious Diseases, 38, S376-S387. doi:10.1086/421400.

Altice, F. L., Mostashari, F., \& Friedland, G. H. (2001). Trust and the acceptance of and adherence to antiretroviral therapy. Journal of Acquired Immune Deficiency Syndromes, 28, 47-58.

Altman, D. G., Schulz, K. F., Moher, D., Egger, M., Davidoff, F., Elbourne, D., et al. (2001). The revised CONSORT statement for reporting randomized trials: Explanation and elaboration. Annals of Internal Medicine, 134, 663-694.

Arnsten, J. H., Demas, P. A., Farzadegan, H., Grant, R. W., Gourevitch, M. N., Chang, C. J., et al. (2001). Antiretroviral therapy adherence and viral suppression in HIV-infected DU: Comparison of self-report and electronic monitoring. Clinical Infectious Diseases, 33, 1417-1423. doi:10.1086/323201.

Arnsten, J. H., Li, X., Mizuno, Y., Knowlton, A. R., Gourevitch, M. N., Handley, K., et al. (2007). Factors associated with antiretroviral therapy adherence and medication errors among HIVinfected injection drug users. Journal of Acquired Immune Deficiency Syndromes, 46, S64-S71.

Atkinson, J. S., Schönnesson, L. N., Williams, M. L., \& Timpson, S. C. (2008). Associations among correlates of schedule adherence to antiretroviral therapy (ART): A path analysis of a sample of crack cocaine using sexually active African-Americans with HIV infection. AIDS Care, 20, 253-262. doi:10.1080/095401207015 06788.

Avants, S. K., Margolin, A., Warburton, L. A., Hawkins, K. A., \& Shi, J. (2001). Predictors of nonadherence to HIV-related medication regimens during methadone stabilization. The American Journal on Addictions, 10, 69-78. doi:10.1080/105504901 750160501.

Bangsberg, D. R. (2006). Less than $95 \%$ adherence to nonnucleoside reverse-transcriptase inhibitor therapy can lead to viral suppression. Clinical Infectious Diseases, 43, 939-941. doi:10.1086/ 507526.

Bassetti, S., Battegay, M., Furrer, H., Rickenbach, M., Flepp, M., Kaiser, L., et al. (1999). Why is highly active antiretroviral therapy (HAART) not prescribed or discontinued? Swiss HIV cohort study. Journal of Acquired Immune Deficiency Syndromes, 21, 114-119.

Berlin, J. A. (1995). Invited commentary. American Journal of Epidemiology, 142, 383-387.

Bouhnik, A. D., Chesney, M., Carrieri, P., Gallais, H., Moreau, J., Moatti, J. P., et al. (2002). Nonadherence among HIV-infected injecting DU: The impact of social instability. Journal of Acquired Immune Deficiency Syndromes, 31, S149-S153.

Bouhnik, A. D., Preau, M., Vincent, E., Carrieri, M. P., Gallais, H., Lepeu, G., et al. (2005). Depression and clinical progression in HIV-infected DU treated with highly active antiretroviral therapy. Antiviral Therapy, 10, 53-61.

Carrieri, M. P., Chesney, M. A., Spire, B., Loundou, A., Sobel, A., Lepeu, G., et al. (2003a). Failure to maintain adherence to HAART in a cohort of French HIV-positive injecting DU. International Journal of Behavioral Medicine, 10, 1-14. doi:10.1207/S15327558IJBM1001_01.
Carrieri, M. P., Raffi, F., Lewden, C., Sobel, A., Michelet, C., Cailleton, V., et al. (2003b). Impact of early versus late adherence to highly active antiretroviral therapy on immunovirological response: A 3-year follow-up study. Antiviral Therapy, 8, 585-594.

Celentano, D. D., Galai, N., Sethi, A. K., Shah, N. G., Strathdee, S. A., Vlahov, D., et al. (2001). Time to initiating highly active antiretroviral therapy among HIV-infected injection DU. AIDS (London, England), 15, 1707-1715.

Celentano, D. D., Vlahov, D., Cohn, S., Shadle, V. M., Obasanjo, O., \& Moore, R. D. (1998). Self-reported antiretroviral therapy in injection DU. JAMA: The Journal of the American Medical Association, 280, 544-546. doi:10.1001/jama.280.6.544.

Chesney, M. A. (2006). The Elusive Gold Standard: Future Perspectives for HIV Adherence Assessment and Intervention. Journal of Acquired Immune Deficiency Syndromes, 43, S149-S155. doi:10.1097/01.qai.0000243112.91293.26.

Chesney, M. A., Ickovics, J. R., Chambers, D. B., Gifford, A. L., Neidig, J., Zwickl, B., et al. (2000). Self-reported adherence to antiretroviral medications among participants in HIV clinical trials: The AACTG adherence instruments. Patient Care Committee \& Adherence Working Group of the Outcomes Committee of the Adult AIDS Clinical Trials Group (AACTG). AIDS Care, 12, 255-266.

Clarke, S., Delamere, S., McCullough, L., Hopkins, S., Bergin, C., \& Mulcahy, F. (2003). Assessing limiting factors to the acceptance of antiretroviral therapy in a large cohort of injecting DU. HIV Medicine, 4, 33-37. doi:10.1046/j.1468-1293.2003.00130.x.

Cofrancesco, J., Jr., Scherzer, R., Tien, P. C., Gibert, C. L., Southwell, H., Sidney, S., et al. (2008). Illicit drug use and HIV treatment outcomes in a US cohort. AIDS (London, England), 22, 357-365.

Cooper, H., \& Hedges, L. V. (1994). The handbook of research synthesis. New York: Russell Sage Foundation.

Crisp, B. R., Williams, M., Timpson, S., \& Ross, M. W. (2004). Medication compliance and satisfaction with treatment for HIV disease in a sample of African-American crack cocaine smokers. AIDS and Behavior, 8, 199-206. doi:10.1023/B:AIBE.0000030 250.33931.af.

Crystal, S., Sambamoorthi, U., Moynihan, P. J., \& McSpiritt, E. (2001). Initiation and continuation of newer antiretroviral treatments among medicaid recipients with AIDS. Journal of General Internal Medicine, 16, 850-859. doi:10.1046/j.15251497.2001.01025.x.

DerSimonian, R., \& Laird, N. (1986). Meta-analysis in clinical trials. Controlled Clinical Trials, 7, 177-188. doi:10.1016/0197-2456 (86)90046-2.

Des Jarlais, D. C., Lyles, C., \& Crepaz, N. (2004). Improving the reporting quality of nonrandomized evaluations of behavioral and public health interventions: The TREND statement. American Journal of Public Health, 94, 361-366.

Duran, S., Solas, C., Spire, B., Carrieri, M. P., Fuzibet, J. G., Costagliola, D., et al. (2001). Do HIV-infected injecting DU over-report adherence to highly active antiretroviral therapy? A comparison between patients' self-reports and serum protease inhibitor concentrations in the French Manif 2000 cohort study. AIDS (London, England), 15, 1075-1077. doi:10.1097/000020 30-200105250-00024.

Egger, M., May, M., Chene, G., Phillips, A. N., Ledergerber, B., Dabis, F., et al. (2002). Prognosis of HIV-1-infected patients starting highly active antiretroviral therapy: A collaborative analysis of prospective studies. Lancet, 360, 119-129. doi: 10.1016/S0140-6736(02)09411-4.

Escobar, I., Campo, M., Martin, J., Fernandez-Shaw, C., Pulido, F., \& Rubio, R. (2003). Factors affecting patient adherence to highly 
active antiretroviral therapy. The Annals of Pharmacotherapy, 37, 775-781. doi:10.1345/aph.1C427.

Fleiss, J. L. (1993). The statistical basis of meta-analysis. Statistical Methods in Medical Research, 2, 121-145. doi:10.1177/0962280 29300200202.

Friedland, G., \& Williams, A. (1999). Attaining higher goals in HIV treatment: The central importance of adherence. AIDS (London, England), 13(Suppl 1), S61-S72.

Gordillo, V., del Amo, J., Soriano, V., \& Gonzalez-Lahoz, J. (1999). Sociodemographic and psychological variables influencing adherence to antiretroviral therapy. AIDS (London, England), 13, 1763-1769. doi:10.1097/00002030-199909100-00021.

Haug, N. A., Sorensen, J. L., Lollo, N. D., Gruber, V. A., Delucchi, K. L., \& Hall, S. M. (2005). Gender differences among HIVpositive methadone maintenance patients enrolled in a medication adherence trial. AIDS Care, 17, 1022-1029. doi:10.1080/ 09540120500100882.

Hedges, L. V., \& Vevea, J. L. (1998). Fixed and random effects models in meta-analysis. Psychological Methods, 3, 486-504. doi:10.1037/1082-989X.3.4.486.

Higgins, J. P., \& Thompson, S. G. (2002). Quantifying heterogeneity in a meta-analysis. Statistics in Medicine, 21, 1539-1558. doi:10.1002/sim.1186.

Higgins, J. P., Thompson, S. G., Deeks, J. J., \& Altman, D. G. (2003). Measuring inconsistency in meta-analyses. BMJ (Clinical Research Ed.), 327, 557-560. doi:10.1136/bmj.327.7414.557.

Hinkin, C. H., Barclay, T. R., Castellon, S. A., Levine, A. J., Durvasula, R. S., Marion, S. D., et al. (2007). Drug use and medication adherence among HIV-1 infected individuals. AIDS and Behavior, 11, 185-194. doi:10.1007/s10461-006-9152-0.

Hogg, R. S., Heath, K. V., Yip, B., Craib, K. J., O'Shaughnessy, M. V., Schechter, M. T., et al. (1998). Improved survival among HIV-infected individuals following initiation of antiretroviral therapy. JAMA: The Journal of the American Medical Association, 279, 450-454. doi:10.1001/jama.279.6.450.

Howard, A. A., Arnsten, J. H., Vlahov, D., Rich, J. D., Schuman, P., Stone, V. E., et al. (2002). A prospective study of adherence and viral load in a large multi-center cohort of HIV-infected women. AIDS (London, England), 16, 2175-2182. doi:10.1097/000020 30-200211080-00010.

Huedo-Medina, T. B., Sánchez-Meca, J., Marín-Martínez, F., \& Botella, J. (2006). Assessing heterogeneity in meta-analysis: Q statistic or I2 index? Psychological Methods, 11, 193-206. doi:10.1037/1082-989X.11.2.193.

Inciardi, J. A., \& Surratt, H. L. (2001). Cross-cultural approaches to harm reduction research: Some considerations on the Brazil experience. Substance Use \& Misuse, 36, 201-212.

Kerr, T., Marshall, A., Walsh, J., Palepu, A., Tyndall, M., Montaner, J., et al. (2005). Determinants of HAART discontinuation among injection drug users. AIDS Care, 17, 539-549.

Kerr, T., Palepu, A., Barness, G., Walsh, J., Hogg, R., Montaner, J., et al. (2004). Psychosocial determinants of adherence to highly active antiretroviral therapy among injection drug users in Vancouver. Antiviral Therapy, 9, 407-414.

Knowlton, A., Arnsten, J., Eldred, L., Wilkinson, J., Gourevitch, M., Shade, S., et al. (2006). Individual, interpersonal, and structural correlates of effective HAART use among urban active injection DU. Journal of Acquired Immune Deficiency Syndromes, 41, 486-492. doi:10.1097/01.qai.0000186392.26334.e3.

Latkin, C. A., Knowlton, A. R., Sherman, S. (2001). Routes of drug administration, differential affiliation, and lifestyle stability among cocaine and opiate users: Implications to HIV prevention. Journal of Substance Abuse, 13, 89-102.

Lipsey, M. W., \& Wilson, D. B. (2001). Practical meta-analysis. Thousand Oaks: Sage.
Liu, H., Longshore, D., Williams, J. K., Rivkin, I., Loeb, T., Warda, U. S., et al. (2006). Substance abuse and medication adherence among HIV-positive women with histories of child sexual abuse. AIDS and Behavior, 10, 279-286. doi:10.1007/s10461-0059041-y.

Lu, M., Safren, S. A., Skolnik, P. R., Rogers, W. H., Coady, W., Hardy, H., et al. (2008). Optimal recall period and response task for self-reported HIV medication adherence. AIDS and Behavior, 12, 86-94. doi:10.1007/s10461-007-9261-4.

Lucas, G. M., Cheever, L. W., Chaisson, R. E., \& Moore, R. D. (2001). Detrimental effects of continued illicit drug use on the treatment of HIV-1 infection. Journal of Acquired Immune Deficiency Syndromes, 27, 251-259.

Lucas, G. M., Gebo, K. A., Chaisson, R. E., \& Moore, R. D. (2002). Longitudinal assessment of the effects of drug and alcohol abuse on HIV-1 treatment outcomes in an urban clinic. AIDS (London, England), 16, 767-774. doi:10.1097/00002030-200203290-00 012.

Malta, M., Strathdee, S. A., Magnanini, M. M. F., \& Bastos, F. I. (2008). Adherence to antiretroviral therapy for HIV/AIDS among drug users-a systematic review. Addiction (Abingdon, England), 103, 1242-1257. doi:10.1111/j.1360-0443.2008.02269.x.

Martin, L. L., Hermandez, S. O., \& Carrobles, J. A. (2005). Psychosocial variables in the antiretroviral treatment adherence in methadone maintenance patients [Article in Spanish]. Psicothema, 17, 575-581.

Martini, M., Recchia, E., Nasta, P., Castanotto, D., Chiaffarino, F., Parazzini, F., et al. (2004). Illicit drug use: Can it predict adherence to antiretroviral therapy? European Journal of Epidemiology, 19, 585-587. doi:10.1023/B:EJEP.0000032353. 03967.ef.

Martín-Sánchez, V., Ortega-Valín, L., Pérez-Simón Mdel, R., Mostaza-Fernández, J. L., Ortiz de Urbina-González, J. J., RodríguezMaría, M., et al. (2002). Factors predicting lack of adherence to highly active antiretroviral treatment. Enfermedades Infecciosas y Microbiología Clinica, 20, 491-497. [Article in Spanish].

Mathers, B. M., Degenhardt, L., Phillips, B., Wiessing, L., Hickman, M., Strathdee, S. A., et al. (2008). Global epidemiology of injecting drug use and HIV among people who inject drugs: A systematic review. The Lancet, 372(9651), 1733-1745. doi:10.1016/S0140-6736(08)61311-2.

McNabb, J., Ross, J. W., Abriola, K., Turley, C., Nightingale, C. H., \& Nicolau, D. P. (2001). Adherence to highly active antiretroviral therapy predicts virologic outcome at an inner-city human immunodeficiency virus clinic. Clinical Infectious Diseases, 33, 700-705. doi:10.1086/322590.

Miller, M. F., Haley, C., Koziel, M. J., \& Rowley, C. F. (2005). Impact of hepatitis $\mathrm{C}$ virus on immune restoration in HIVinfected patients who start highly active antiretroviral therapy: A meta-analysis. Clinical Infectious Diseases, 41, 713-720. doi: $10.1086 / 432618$.

Mills, E. J., Nachega, J. B., Buchan, I., Orbinski, J., Attaran, A., Singh, S., et al. (2006). Adherence to antiretroviral therapy in sub-Saharan Africa and North America: A meta-analysis. JAMA : The Journal of the American Medical Association, 296, 679690. doi:10.1001/jama.296.6.679.

Moatti, J. P., Carrieri, M. P., Spire, B., Gastaut, J. A., Cassuto, J. P., \& Moreau, J. (2000). Adherence to HAART in French HIVinfected injecting DU: The contribution of buprenorphine drug maintenance treatment. The Manif 2000 study group. AIDS (London, England), 14, 151-155. doi:10.1097/00002030200001280-00010.

Moher, D., Coo, D. J., Eastwood, S., Olin, I., Rennie, D., \& Stroup, D. F. (2000). Improving the quality of reports of meta-analysis of randomized controlled trials: The QUOROM Statement. British 
Journal of Surgery, 87, 1448-1454. doi:10.1046/j.13652168.2000.01610.x

Moher, D., Schulz, K. F., \& Altman, D. G. (2001). The CONSORT statement: Revised recommendations for improving the quality of reports of parallel-group randomised trials. Lancet, 357, 1191-1194. doi:10.1016/S0140-6736(00)04337-3.

New York State Department of Health. (2005). Adherence to antiretroviral therapy among substance users. New York (NY): New York State Department of Health.

Palella, F. J., Jr, Delaney, K. M., Moorman, A. C., Loveless, M. O., Fuhrer, J., Satten, G. A., et al. (1998). Declining morbidity and mortality among patients with advanced human immunodeficiency virus infection. HIV Outpatient Study Investigators. The New England Journal of Medicine, 338, 853-860. doi:10.1056/ NEJM199803263381301.

Palepu, A., Horton, N. J., Tibbetts, N., Meli, S., \& Samet, J. H. (2004a). Uptake and adherence to highly active antiretroviral therapy among HIV-infected people with alcohol and other substance use problems: The impact of substance abuse treatment. Addiction (Abingdon, England), 99, 361-368. doi:10.1111/j.1360-0443.2003.00670.x.

Palepu, A., Tyndall, M. W., Chan, K., Wood, E., Montaner, J. S., \& Hogg, R. S. (2004b). Initiating highly active antiretroviral therapy and continuity of HIV care: The impact of incarceration and prison release on adherence and HIV treatment outcomes. Antiviral Therapy, 9, 713-719.

Palepu, A., Tyndall, M. W., Joy, R., Kerr, T., Wood, E., Press, N., et al. (2006). Antiretroviral adherence and HIV treatment outcomes among HIV/HCV co-infected injection DU: The role of methadone maintenance therapy. Drug and Alcohol Dependence, 84, 188-194. doi:10.1016/j.drugalcdep.2006.02.003.

Palepu, A., Tyndall, M. W., Li, K., Yip, B., O’Shaughnessy, M. V., Schechter, M. T., et al. (2003a). Alcohol use and incarceration adversely affect HIV-1 RNA suppression among injection DU starting antiretroviral therapy. Journal of Urban Health, 80, 667-675. doi:10.1093/jurban/jtg073.

Palepu, A., Tyndall, M., Yip, B., O'Shaughnessy, M. V., Hogg, R. S., \& Montaner, J. S. (2003b). Impaired virologic response to highly active antiretroviral therapy associated with ongoing injection drug use. Journal of Acquired Immune Deficiency Syndromes, 32, 522-526. doi:10.1097/00126334-200304150-00009.

Parruti, G., Manzoli, L., Toro, P. M., D’Amico, G., Rotolo, S., Graziani, V., et al. (2006). Long-term adherence to first-line highly active antiretroviral therapy in a hospital-based cohort: Predictors and impact on virologic response and relapse. AIDS Patient Care and STDs, 20, 48-56.

Pearson, C. R., Micek, M. A., Simoni, J. M., Hoff, P. D., Matediana, E., Martin, D. P., et al. (2007a). Randomized control trial of peer-delivered, modified directly observed therapy for HAART in Mozambique. Journal of Acquired Immune Deficiency Syndromes, 46, 238-244. doi:10.1097/QAI.0b013e318153f7ba.

Pearson, C. R., Simoni, J. M., Hoff, P., Kurth, A. E., \& Martin, D. P. (2007b). Assessing antiretroviral adherence via electronic drug monitoring and self-report: An examination of key methodological issues. AIDS and Behavior, 11, 161-173. doi:10.1007/ s10461-006-9133-3.

Pinheiro, C., De-Carvalho-Leite, J., Drachler, M., \& Silveira, V. L. (2002). Factors associated with adherence to antiretroviral therapy in HIV/AIDS patients: A cross-sectional study in southern Brazil. Brazilian Journal of Medical and Biological Research, 35, 1173-1181.

Pradier, C., Carrieri, P., Bentz, L., Spire, B., Dellamonica, P., Moreau, J., et al. (2001). Impact of short-term adherence on virological and immunological success of HAART: A case study among French HIV-infected IDU. International Journal of STD and AIDS, 12, 324-328. doi:10.1258/0956462011923165.
Purcell, D. W., Metsch, L. R., Latka, M., Santibanez, S., Gomez, C. A., Eldred, L., et al. (2004). Interventions for seropositive injectors-research and evaluation: An integrated behavioral intervention with HIV-positive injection DU to address medical care, adherence, and risk reduction. Journal of Acquired Immune Deficiency Syndromes, 37, S110-S118. doi:10.1097/01.qai.0000 140609.44016.c4.

Quinn, T. C., Wawer, M. J., Sewankambo, N., Serwadda, D., Li, C., Wabwire-Mangen, F., et al. (2000). Viral load and heterosexual transmission of human immunodeficiency virus type 1. Rakai Project Study Group. The New England Journal of Medicine, 342, 921-929. doi:10.1056/NEJM200003303421303.

R Core Development Core Team. (2004). R: A language and environment for statistical computing. Vienna, Austria: R-Foundation for Statistical Computing. Retrieved June 18, 2008, from: http://www.R-project.org.

Roca, B., Gomez, C. J., \& Arnedo, A. (1999). Stavudine, lamivudine and indinavir in drug abusing and non-drug abusing HIVinfected patients: Adherence, side effects and efficacy. The Journal of Infection, 39, 141-145. doi:10.1016/S0163-4453(99) 90006-3.

Sharpe, T. T., Lee, L. M., Nakashima, A. K., Elam-Evans, L. D., \& Fleming, P. L. (2004). Crack cocaine use and adherence to antiretroviral treatment among HIV-infected black women. Journal of Community Health, 29, 117-127. doi:10.1023/ B:JOHE.0000016716.99847.9b.

Simoni, J. M., Pantalone, D. W., Plummer, M. D., \& Huang, B. (2007). A randomized controlled trial of a peer support intervention targeting antiretroviral medication adherence and depressive symptomatology in HIV-positive men and women. Health Psychology, 26, 488-495. doi:10.1037/0278-6133.26.4.488.

Smith-Rohrberg, D., Mezger, J., Walton, M., Bruce, R. D., \& Altice, F. L. (2006). Impact of Enhanced Services on Virologic Outcomes in a Directly Administered Antiretroviral Therapy Trial for HIV-Infected DU. Journal of Acquired Immune Deficiency Syndromes, 43, S48-S53. doi:10.1097/01.qai.0000 248338.74943.85.

Strathdee, S. A., Palepu, A., Cornelisse, P. G., Yip, B., O’Shaughnessy, M. V., Montaner, J. S., et al. (1998). Barriers to use of free antiretroviral therapy in injection drug users. JAMA : The Journal of the American Medical Association, 280, 547-549. doi:10.1001/jama.280.6.547.

Stroup, D. F., Berlin, J. A., Morton, S. C., Olkin, I., Williamson, G. D., Rennie, D., et al. (2000). Meta-analysis of observational studies in epidemiology: A proposal for reporting. JAMA : The Journal of the American Medical Association, 283, 2008-2012. doi:10.1001/jama.283.15.2008.

Turner, B. J., Laine, C., Cosler, L., \& Hauck, W. W. (2003). Relationship of gender, depression, and health care delivery with antiretroviral adherence in HIV-infected DU. Journal of General Internal Medicine, 18, 248-257. doi:10.1046/j.1525-1497.2003. 20122.x

van Houwelingen, H. C., Arends, L. R., \& Stijnen, T. H. (2002). Tutorial in biostatistics. Advanced methods in meta-analysis: Multivariate approach and meta-regression. Statistics in Medicine, 21, 589-624. doi:10.1002/sim.1040.

Viechtbauer, W. (2007). MiMa: An S-Plus/R function to fit metaanalytic mixed-, random-, and fixed-effects models. http:// www.wvbauer.com/downloads/mima.ssc. Retrieved 18 June 2008.

Vissoci Reiche, E. M., Bonametti, A. M., Morimoto, H. K., Morimoto, A. A., Wiechemann, S. L., Matsuo, T., et al. (2008). Epidemiological, immunological and virological characteristics, and disease progression of HIV-1/HCV-co-infectedc patients from a southern Brazilian population. International Journal of Molecular Medicine, 21, 387-395. 
Vlahov, D., \& Celentano, D. D. (2006). Access to highly active antiretroviral therapy for injection DU: Adherence, resistance, and death. Cadernos de Saude Publica, 22, 705-718.

Wainberg, M. A., \& Friedland, G. (1998). Public health implications of antiretroviral therapy and HIV drug resistance. JAMA: The Journal of the American Medical Association, 279, 1977-1983.

Waldrop-Valverde, D., Ownby, R. L., Wilkie, F. L., Mack, A., Kumar, M., \& Metsch, L. (2006). Neurocognitive aspects of medication adherence in HIV-positive injecting DU. AIDS and Behavior, 10, 287-297. doi:10.1007/s10461-005-9062-6.

Waldrop-Valverde, D., \& Valverde, E. (2005). Homelessness and psychological distress as contributors to antiretroviral nonadherence in HIV-positive injecting DU. AIDS Patient Care and STDs, 19, 326-334. doi:10.1089/apc.2005.19.326.

Wang, C., Vlahov, D., Galai, N., Bareta, J., Strathdee, S. A., Nelson, K. E., et al. (2004). Mortality in HIV-seropositive versus seronegative persons in the era of highly active antiretroviral therapy: Implications for when to initiate therapy. The Journal of Infectious Diseases, 190, 1046-1054. doi:10.1086/422848.

Wensing, A. M., van de Vijver, D. A., Angarano, G., Asjö, B., Balotta, C., Boeri, E., et al. (2005). Prevalence of drug-resistant HIV-1 variants in untreated individuals in Europe: Implications for clinical management. The Journal of Infectious Diseases, 192, 958-966.

WHO/UNAIDS. (2007). Report on the global AIDS epidemic. Genebra: UNAIDS and WHO, 2007.
Wood, E., Hogg, R. S., Lima, V. D., Kerr, T., Yip, B., Marshall, B. D., et al. (2008). Highly active antiretroviral therapy and survival in HIV-infected injection drug users. JAMA: The Journal of the American Medical Association, 300, 550-554. doi:10.1001/ jama.300.5.550.

Wood, E., Hogg, R. S., Yip, B., Harrigan, P. R., O’Shaughnessy, M. V., \& Montaner, J. S. (2004a). The impact of adherence on CD4 cell count responses among HIV-infected patients. Journal of Acquired Immune Deficiency Syndromes, 35, 261-268. doi:10.1097/00126334-200403010-00006.

Wood, E., Montaner, J. S., Bangsberg, D. R., Tyndall, M. W., Strathdee, S. A., O'Shaughnessy, M. V., et al. (2003a). Expanding access to HIV antiretroviral therapy to marginalised populations in the developed world. AIDS (London, England), 17, 2419-2427. doi:10.1097/00002030-200311210-00003.

Wood, E., Montaner, J. S., Yip, B., Tyndall, M. W., Schechter, M. T., O'Shaughnessy, M. V., et al. (2003b). Adherence and plasma HIV RNA responses to highly active antiretroviral therapy among HIV-1 infected injection DU. Canadian Medical Association Journal, 169, 656-661.

Wood, E., Montaner, J. S., Yip, B., Tyndall, M. W., Schechter, M. T., O'Shaughnessy, M. V., et al. (2004b). Adherence to antiretroviral therapy and CD4 T-cell count responses among HIVinfected injection drug users. Antiviral Therapy, 9, 229-235. 